EUROPHYSICS LETTERS

Europhys. Lett., (), pp. ()

\title{
Dynamics of Strongly Deformed Polymers in Solution
}

\author{
Roland Rzehak and Walter Zimmermann \\ Theoretische Physik, Universität des Saarlandes, D-66041 Saarbrücken \\ Institut für Festkörperforschung and FORUM, Forschungszentrum Jülich, D-52425 \\ Jülich \\ (received ; accepted ) \\ PACS. 83.10.Mj- Molecular dynamics, Brownian dynamics . \\ PACS. 36.20.Ey - Conformation (Statistics and Dynamics). \\ PACS. 47.+d - Non-Newtonian fluid flows.
}

\begin{abstract}
Bead spring models for polymers in solution are nonlinear if either the finite extensibility of the polymer, excluded volume effects or hydrodynamic interactions between polymer segments are taken into account. For such models we use a powerful method for the determination of the complete relaxation spectrum of fluctuations at steady state. In general, the spectrum and modes differ significantly from those of the linear Rouse model. For a tethered polymer in uniform flow the differences are mainly caused by an inhomogeneous distribution of tension along the chain and are most pronounced due to the finite chain extensibility. Beyond the dynamics of steady state fluctuations we also investigate the nonlinear response of the polymer to a large sudden change in the flow. This response exhibits several distinct regimes with characteristic decay laws and shows features which are beyond the scope of single mode theories such as the dumbbell model.
\end{abstract}

Introduction. - The slow internal dynamics of polymers makes polymer solutions viscoelastic which is manifest in such spectacular effects as turbulent drag reduction. In spite of active research for more than 60 years already, the understanding of these phenomena is still incomplete $[1,2,3,4,5,6,7,8]$. Progress towards a microscopically founded rather than empirical description of flowing polymer solutions is possible only by a thorough analysis of the interplay between single polymers and the flow. However, only recent experiments on individual DNA molecules allow to observe the molecular conformations $[9,10,11,12]$ and computer simulations facilitate a treatment of the nonlinear interactions within the polymer-solvent system $[13,14,15,16,17]$.

The dynamics of fluctuations of a polymer in flow is commonly characterized by relaxation times and modes, which also determine the linear viscoelastic properties. These quantities are known analytically only for the linear Rouse and Zimm models [5, 6]. Questions arising are: Is there an effective method for determining the complete relaxation spectrum and the corresponding modes for fluctuations around an arbitrary stationary state also for more realistic nonlinear polymer models? What is their dependence on the polymer deformation and which decay laws describe the whole relaxation process of a strongly stretched polymer to

Typeset using EURO-TEX 
equilibrium?

Here we give answers for the model problem of a tethered polymer in uniform flow which has been much investigated recently both experimentally $[9,10]$ and theoretically using blob models $[18,15]$ and bead spring models $[13,14,15,16,17]$. By simulation of bead spring chains we determine the relative importance for the polymer dynamics of various interactions such as finite extensibility, excluded volume interactions (EVI), and hydrodynamic interactions (HI). Due to Brownian motion, even in a steady state the beads fluctuate around some timeindependent average positions. In the equilibrium state of a tethered polymer these average positions all coincide with the tether-point, while if the polymer is stretched by a flow or force they assume non-trivial values. Our analysis of the conformational fluctuations in such a statistically steady state is based on the covariance matrix of bead positions. This approach yields the full relaxation spectrum rather than only the longest relaxation time considered in previous works and it provides numeric values for the relaxation times which cannot be obtained from common scaling arguments. Furthermore it gives a corresponding complete set of uncorrelated relaxation modes and thus constitutes a generalization of the normal mode analysis used for the linear models of Rouse and Zimm which is applicable to nonlinear polymer models.

Theories of polymer deformation in simple shear or elongational flows rely on the so-called time criterion [7]: the polymer will be significantly deformed when the inverse shear or elongation rate $\kappa^{-1}$ is shorter than the longest polymer relaxation time. Since the latter is conformationdependent because of hydrodynamic backflow, it was argued that a hysteretic transition between a coiled and a stretched polymer conformation may occur upon varying $\kappa[7,8]$. Implicit in the use of the time criterion is the assumption that the polymer is in the steady state corresponding to the local flow field at any time. This requires variations in the flow field experienced by the polymer to be slow - an assumption which is probably not valid in flows as they occur e.g. in turbulent drag reduction. An interesting model for such rapidly varying flows is provided by the immediate start-up and cessation of simple flows like the uniform flow acting on a tethered polymer considered here. For this case we identify several regimes governed by different decay laws which have not been described before. This complex behavior cannot be captured by a simple dumbbell model and it coincides with the relaxation of fluctuations in the steady state only in the linear response regime of polymer dynamics.

Bead Spring Polymer Model. - In our simulations we follow the Brownian dynamics of a bead spring model as described in more detail in Refs. [16, 17]. The equation of motion for the vector $\mathbf{R}=\left(\mathbf{R}_{1}, \ldots, \mathbf{R}_{N}\right)$ of all bead positions may be written in the form

$$
\dot{\mathbf{R}}=\mathbf{v}+\mathbf{H} \mathbf{F}+\sqrt{2 k_{B} T \mathbf{H}} \boldsymbol{\xi} .
$$

Here $\mathbf{v}=v \hat{\mathbf{x}}$ is the velocity of the unperturbed flow, $k_{B}$ is the Boltzmann constant, $T$ the temperature and $\xi(t)$ is an uncorrelated Gaussian white noise with zero mean and unit variance. The potential forces $\mathbf{F}$ comprise a repulsive Lennard Jones force describing the excluded volume interactions (EVI) and the next-neighbor bond forces for which we use either a linear or the familiar FENE (Finite Extensible Nonlinear Elastic) force law. The latter provides a reasonable approximation of polymers with a fixed bond length for flow velocities $v \leq 0.5$. Throughout this work we use a chain length of $N=100$ and the parameters appearing in the potential forces $\mathbf{F}$ are the same as in Ref. [19], where this choice was shown to prohibit bond crossings in the case with EVI. The hydrodynamic interactions (HI) are incorporated in the Oseen tensor approximation [3], where the flow perturbations due to all beads simply superimpose and the additional drag forces can be expressed by the off-diagonal part of the mobility tensor $\mathbf{H}$. We have $\mathbf{H}_{i j}=\Omega\left(\mathbf{R}_{i}-\mathbf{R}_{j}\right)$ for $i \neq j$ with $\Omega(\mathbf{r})=\left(\mathbf{1}+\hat{\mathbf{r}} \hat{\mathbf{r}}^{T}\right) /(8 \pi \eta|\mathbf{r}|)$ and 
$\mathbf{H}_{i i}=\mathbf{1} / \zeta$ with $\zeta=6 \pi \eta a$ the friction coefficient of a bead with radius $a$ in a solvent of viscosity $\eta$. In contrast to previous work $[13,14]$ we do not average $\mathbf{H}$ which would effectively linearize the equation of motion. Since the Oseen tensor becomes non-positive for bead separations of the order of $a$, we always consider HI together with EVI. The values $\eta=0.2, \zeta=1.0$, $k_{B} T=1.0$ and $b=1.0$ (harmonic springs) or $b=0.961$ (FENE springs) for the bond length $b$ fix the strength of the HI and the units of force, energy, length etc. .

Covariance Matrix. - Analytically the polymer relaxation spectrum and modes can be calculated only for the Rouse and Zimm models $[5,6]$ which are governed by linear equations of motion by virtue of the assumptions made. For realistic polymer models in contrast, the equation of motion, as described above, is nonlinear.

Nevertheless, the relaxation times $\tau_{p}$ of the Rouse amplitudes are often used also for nonlinear polymer models in order to characterize the dynamics of fluctuations in steady states. Each Rouse amplitude is obtained by projecting a time series of polymer conformations on the respective Rouse mode and $\tau_{p}$ is determined by an exponential fit to the time evolution of this amplitude [20]. The determination of $\tau_{p}$ in this way is tedious and error-prone so that in practice only the few longest relaxation times can be obtained. In addition, the significance of the Rouse modes for nonlinear models is unclear. Another approach [9] aims at the relaxation spectrum directly, without referring to corresponding modes, by applying an inverse Laplace transform to a single time series of some observable like the end-to-end distance. Due to the presence of noise in the data this requires the use of special regularization techniques and the results may depend strongly on the regularization parameter.

Here we determine the polymer relaxation spectrum and a corresponding set of uncorrelated modes from the covariance matrix of bead positions. This approach is motivated by a relation between the initial decay-rate of correlations in the dynamics of conformational fluctuations in a steady state and the second moments of the statistics of bead positions. This relation may be proved for arbitrary polymer models in thermal equilibrium, for models without HI, or for a preaveraged mobility tensor $\langle\mathbf{H}\rangle$ by using the the linear response formalism as described e.g. in Ref.[3]. To be specific: A large sample of data is generated by integrating equation (1) numerically over a long time and storing all bead positions in equal time intervals. Then from this time series $\mathbf{x}(\mu)=\mathbf{R}(\mu \cdot \Delta t)$ the mean bead positions $\langle\mathbf{R}\rangle=\sum_{\mu=1}^{M} \mathbf{R}(\mu \cdot \Delta t) / M$ are calculated as well as the covariance matrix $\mathbf{C}$ of the deviations $\mathbf{y}=\mathbf{R}-\langle\mathbf{R}\rangle$

$$
\mathbf{C}=\left\langle\mathbf{y} \mathbf{y}^{T}\right\rangle\left\langle k_{\mathrm{B}} T \mathbf{H}\right\rangle^{-1} .
$$

Once $\mathbf{C}$ is obtained, its eigenvalues $\tau_{p}$ give the complete spectrum of relaxation times of fluctuations in a steady state. The eigenvectors $\mathbf{y}_{p}^{\alpha}$ of $\mathbf{C}, \alpha=x, y, z, p=1 \ldots N$, are uncorrelated and form a basis of the space of polymer conformations such that the deviations $\mathbf{y}$ may be expressed as a linear combination $\mathbf{y}=\sum_{p, \alpha} a_{p}^{\alpha} \mathbf{y}_{p}^{\alpha}$ with amplitudes $a_{p}^{\alpha}$. At equilibrium $\langle\mathbf{R}\rangle=0$ and the modes $\mathbf{y}_{p}^{\alpha}=\mathbf{R}_{p}^{\alpha}$ describe special polymer conformations where the position of the $i$-th bead $\left[\mathbf{R}_{p}^{\alpha}\right]_{i}$ may be factored in a direction $\hat{\mathbf{E}}^{\alpha}$ and a distance function $R_{p i}$ as $\left[\mathbf{R}_{p}^{\alpha}\right]_{i}=R_{p i} \hat{\mathbf{E}}^{\alpha}$. The relaxation spectrum is threefold degenerate due to symmetry. In the presence of a uniform flow one can distinguish one longitudinal mode along the flow direction and two transverse modes perpendicular to it. Since $\langle\mathbf{R}\rangle \neq 0$ away from equilibrium, $\left[\mathbf{y}_{p}^{\alpha}\right]_{i}$ is not the absolute position of bead $i$ when the mode is excited but the deviation from its mean position. The $\left[\mathbf{y}_{p}^{\alpha}\right]_{i}$ are a natural generalization of the Rouse modes for deformed polymers with nonlinear laws of motion. From a practical point of view this method to compute relaxation times is fast, easy to use, needs no adjustments of parameters or additional assumptions, and yields the complete spectrum in one sweep. A more detailed discussion and a possible extension of this method that does not invoke preaveraging of $\mathbf{H}$ will be given elsewhere. 

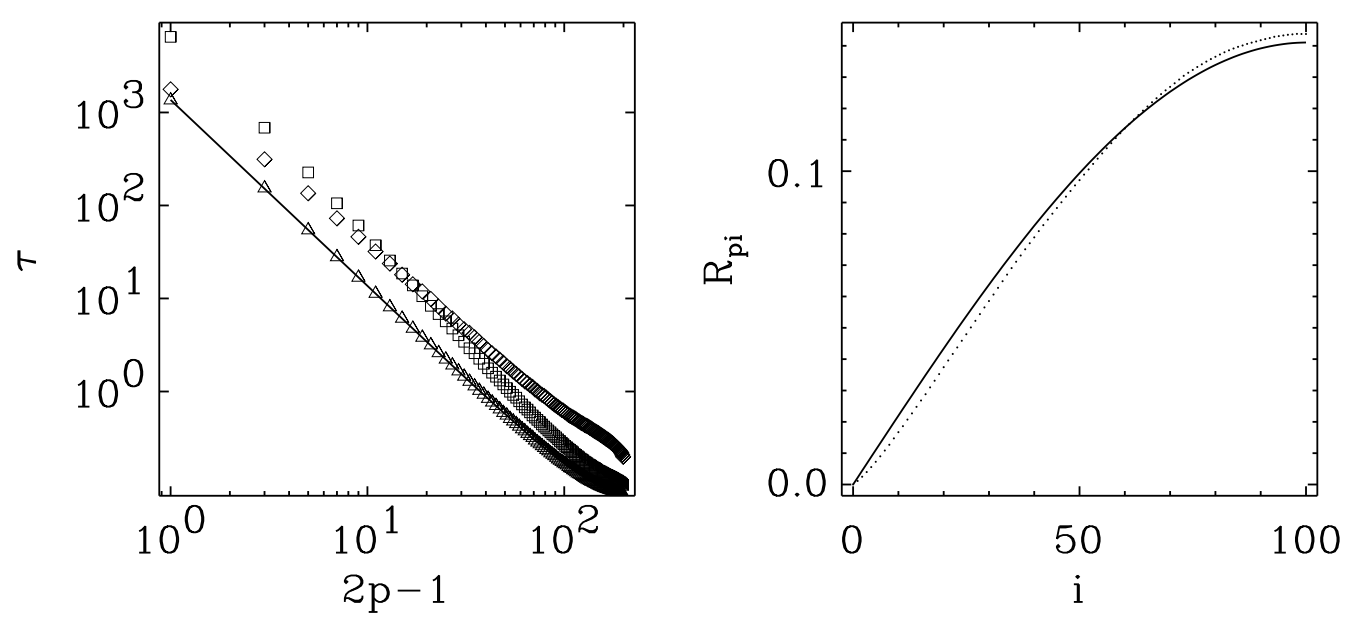

Fig. 1. - Left: Relaxation spectra for the Rouse chain calculated analytically (solid line) and from the matrix C (triangles) compared with the case including EVI (squares) or both EVI and HI (diamonds). Right: First mode $(p=1)$ for a pure Rouse chain (solid line) and with EVI included (dotted line).

Relaxation of Fluctuations. - The relaxation spectrum of a Rouse chain in thermal equilibrium as calculated by the method described above is shown in Fig. 1. It agrees perfectly with the analytical result

$$
\tau_{p}^{R}=\frac{\zeta}{k_{H}}\left(4 \sin ^{2}\left(\frac{2 p-1}{2 N+1} \frac{\pi}{2}\right)\right)^{-1}
$$

where $\zeta$ is the single bead friction coefficient and $k_{\mathrm{H}}$ is the force constant of the springs connecting the beads. The usual scaling $\tau_{p}^{R} \propto(2 p-1)^{-2}[2,3]$ follows for $(2 p-1) / N \rightarrow 0$. Of the nonlinear effects considered here EVI have not been included in previous work concerned with strong deformations of polymers $[7,8,13,14]$. However, a comparison with the Rouse modes in Fig. 1 shows that even in equilibrium EVI leads to a small but significant modification of the relaxation modes. The scaling of the relaxation times in this case is $\tau_{p} \propto(2 p-1)^{-2.2}$. This is precisely the inverse of the power law dependence on the chain length $\tau \propto N^{2 \nu+1}$ predicted by scaling theory $[2,3]$ for a polymer with EVI where $\nu=3 / 5$ which is also reproduced by the analysis given above. In contrast to scaling theory, our method also yields a number for the longest relaxation time $\tau_{1}=6.5 \cdot 10^{3}$ which turns out to be about a factor of 5 larger with than without EVI. Upon addition of HI to the model the properties of the Zimm model $[6,3]$ are reproduced, i.e. $\tau_{p} \propto(2 p-1)^{-1.8}$. Replacing harmonic by FENE springs does not affect the equilibrium relaxation times or modes. Away from equilibrium, however, this has a profound impact. As shown in Fig. 2, the modes are very different from the Rouse form and the relaxation times decrease strongly with increasing flow velocity. A decrease of the relaxation times has been reported recently also for a chain which is pulled at the ends [14]. Experimentally it was observed that the polymer relaxation in this case can be described by the Rouse modes [11]. Pulling a polymer at the ends, however, is special in that the tension along the polymer is everywhere the same. In contrast, when the polymer is stretched by a flow then the tension in general is inhomogeneous [16]. A comparison of the modes calculated for both cases reveals that just this inhomogeneity causes the strong deviation of the modes from the Rouse form as shown in Fig. 2. We remark that such inhomogeneous tension also 

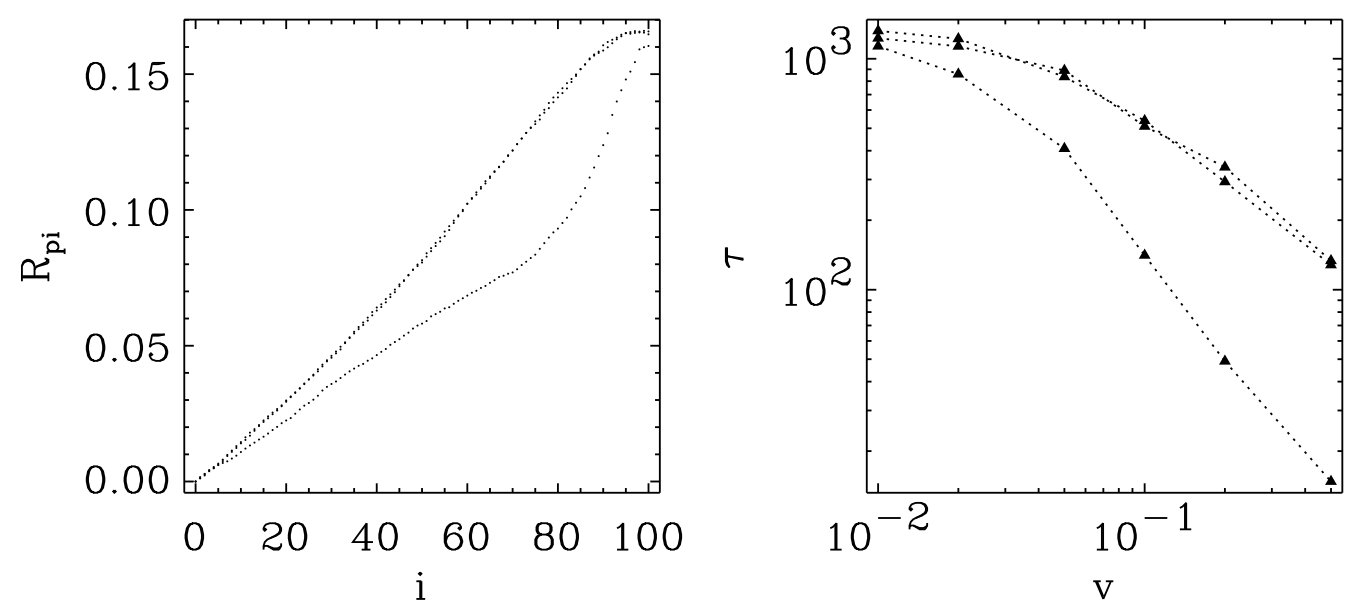

Fig. 2. - Relaxation modes and times for a pure FENE chain. Left: First triplet $(\mathrm{p}=1)$ of relaxation modes for $v=0.2$. Right: Triplet of longest relaxation times as function of the flow velocity $v$ (the dotted lines are only a guide to the eye here). In each figure, the lower line corresponds to the longitudinal mode where $\mathbf{E}^{\alpha}$ is aligned with the flow, while the upper two lines correspond to the two degenerate transverse modes.

arises due to EVI and HI [16].

To identify the effects of HI on the relaxation of a polymer in uniform flow, we compare models with harmonic springs and EVI for the two cases without and with HI. In the first case the relaxation times decrease in stronger flows since EVI are less important for an elongated polymer [16]. For $v \gtrsim 0.2$ the modes assume Rouse shape and both scaling and numerical values of the relaxation spectrum exhibit a crossover to Rouse behavior. Upon adding HI to the model, the relaxation times behave non-monotonic: after an initial decay they increase with $v$ in an intermediate range $0.2 \lesssim v \lesssim 2.0$ and only at very large flow velocities $v>5.0$ there is a crossover to Rouse behavior. For the chain lengths available in the present study, the combination of FENE springs with EVI and HI results in a monotonic decrease of the longest relaxation time.

Average Dynamics. - After cessation of the uniform flow the tethered polymer relaxes from an elongated to a coiled state. For the model with harmonic springs the mean $x$-component of the end-to-end vector $X_{E}(t)$ decays exponentially with a time constant $\tau_{p}^{R}$, cf. Eq. (3). When EVI are added, the polymer relaxation exhibits three distinct regimes as shown in Fig. 3. Initially, $X_{E}(t)$ decays exponentially with time constant $\tau_{1}^{R}$. This regime is dominated by the relaxation of the stretched part of the chain close to the tether-point where EVI are negligible. Then, one finds a stretched exponential decay $X_{E}(t) \propto \exp \left(-\left(t / \tau_{1}^{R}\right)^{\beta}\right)$ with stretching exponent $\beta \simeq 0.5$. Neither simple exponentials nor power laws gave satisfactory agreement with the simulation data. During this stage, the relaxation is increasingly slowed down because self-avoidance constraints become more important. A common explanation of stretched exponential behavior is by a superposition of single exponential decays with time constants that obey a power law. A power law spectrum of modes is provided here by the Rouse modes which all become excited due to the nonlinear coupling by EVI even when initially only the lowest mode is present. It is unlikely that this complex relaxation behavior can be captured by a single mode theory like the dumbbell model. In a third regime finally, which sets in when $X_{E}(t)$ equals 

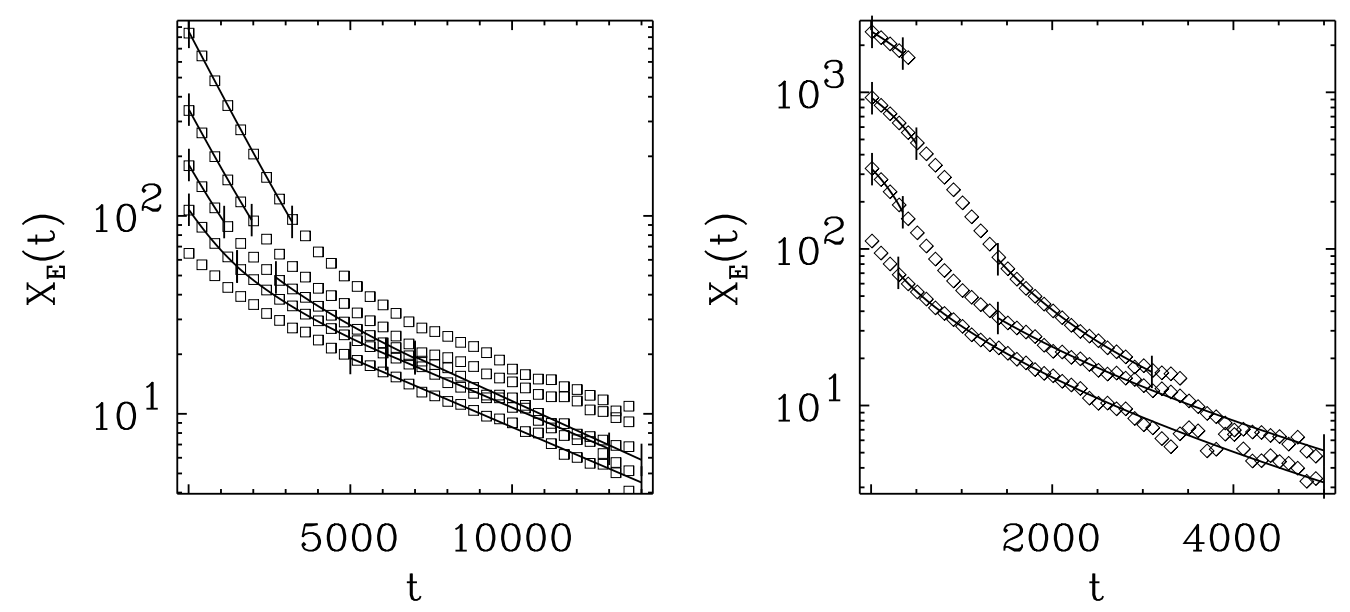

Fig. 3. - Evolution of the $x$-component of the end-to-end vector $X_{E}(t)$ averaged over 100 runs. Left: Rouse chain with EVI after cessation of a flow with $v=0.5,0.2,0.1,0.05,0.02$ from top to bottom. Right: Rouse chain with both EVI and HI after cessation of a flow with $v=2.0,1.0,0.5,0.2$ from top to bottom. Different regimes of the relaxation process are marked by vertical bars on the solid lines representing fits with simple decay laws (see text). Only every 30-th (squares) respectively 10-th (diamonds) point of the data used for the fits is shown.

the equilibrium end-to-end distance $R_{E}=22.2$, the entangling process is completed and the vanishing equilibrium value of $X_{E}$ is approached by only local rearrangements within the coil. This process is again described by a single exponential decay law where the time constant is now given by the longest relaxation time in equilibrium, cf. Fig. 1. When in addition to EVI also $\mathrm{HI}$ are included in the model, a very different behavior results in the initial phase of the relaxation for large velocities $v \geq 0.5$. In this case the polymer at first appears to move freely, i.e. $\partial X_{E} / \partial t \propto v$. This is the consequence of a large flow perturbation due to average forces acting on the beads which leads to a highly cooperative motion. This is very different from the steady state where the flow perturbation is caused only by fluctuating forces. Replacing the harmonic by FENE springs causes a short and rapid initial recoil but the later stages of the relaxation remain qualitatively unaffected.

After the flow is started, simulation data (not shown in the figure) reveal for all models at first a short period of apparent free polymer motion. This is followed by a single exponential growth of $X_{E}$ which is well described by the longest relaxation time in the stretched final state. The pure Rouse model is exceptional since the initial regime is confined to the shortest relaxation time.

Conclusions. - Using the second moments of the statistics of bead positions, we have applied a powerful method for the determination of the complete polymer relaxation spectrum and corresponding modes from simulation data. Our approach works for both nonlinear polymer models and arbitrary steady states as caused by the action of flows or forces on the polymer. For a tethered polymer in uniform flow, the finite extensibility causes a monotonic decrease of relaxation times with increasing elongation. Older theories of the coil stretch transition in elongational flow, in contrast, were based on the assumption of increasing relaxation times [2]. This finding agrees with recent results obtained for a chain pulled only at the ends $[14,11]$ where the modes remain Rouse-like. The modes for a polymer stretched by a flow, in contrast, 
are very different from the Rouse modes because the tension along the polymer then in general is inhomogeneous. On the other hand we found that HI may lead to a non-monotonic velocity dependence of the relaxation times. In order to draw a final conclusion about the occurrence of a coil-stretch transition as predicted for elongational flow, longer chains would have to be considered.

Going beyond the relaxation dynamics of polymers close to steady states, we also dropped the common preaveraging approximation for the mobility tensor. Thus, in contrast to other recent work, all effects relevant for uncharged polymers in flows are covered. Specifically, we have considered the response of a tethered polymer to start-up or cessation of the flow and found that EVI causes a stretched exponential relaxation which can only be described by a model with many degrees of freedom and not by a simple dumbbell model. Scaling relations predicted by common blob models [18] are unlikely to occur in simulations because the blob models do not include inhomogeneous effects of EVI and HI $[15,16]$. A more detailed discussion of relations to other work and an extension of this study to other flow fields will be given elsewhere.

It is a pleasure to acknowledge fruitful discussions with B. Dünweg and D. Kienle.

\section{REFERENCES}

[1] J. D. Ferry, Viscoelastic Properties of Polymers, 3rd ed. (John Wiley, New York, 1980); R. B. Bird, R. C. Armstrong, and O. Hassager, Dynamics of Polymeric Liquids, Vols. I, II, 2nd ed. (John Wiley, New York, 1987).

[2] P.-G. deGennes, Scaling Concepts in Polymer Physics (Cornell University Press, Ithaca NY, 1981).

[3] M. Doi and S. F. Edwards, The Theory of Polymer Dynamics (Clarendon Press, Oxford, 1986).

[4] A. Gyr and H.-W. Bewersdorff, Drag Reduction of Turbulent Flows by Additives (Kluwer, Dordrecht, 1995).

[5] P. E. Rouse, Jr., J. Chem. Phys. 21, 1272 (1953).

[6] B. H. Zimm, J. Chem. Phys. 24, 269 (1956).

[7] P.-G. deGennes, J. Chem. Phys. 60, 5030 (1974).

[8] E. J. Hinch, Phys. Fluids 20, S22 (1977).

[9] T. T. Perkins, S. R. Quake, D. E. Smith, and S. Chu, Science 264, 822 (1994).

[10] S. Manneville et al., Europhys. Lett. 36, 413 (1996).

[11] S. Quake, H. Babcock, and S. Chu, Nature 388, 151 (1997).

[12] T. T. Perkins, D. E. Smith, and S. Chu, Science 276, 2016 (1997). D. E. Smith, H. P. Babcock, and S. Chu, Science 283, 1724 (1999).

[13] R. G. Larson, T. T. Perkins, D. E. Smith, and S. Chu, Phys. Rev. E 55, 1794 (1997).

[14] J. W. Hatfield and S. R. Quake, Phys. Rev. Lett. 82, 3548 (1999).

[15] R. Rzehak, D. Kienle, T. Kawakatsu, and W. Zimmermann, Europhys. Lett. 46, 821 (1999).

[16] R. Rzehak, W. Kromen, T. Kawakatsu, and W. Zimmermann, Eur. Phys. J. E 2, 3-30 (2000).

[17] R. Rzehak, D. Kienle, T. Kawakatsu, and W. Zimmermann in Molecular Dynamics on Parallel Computers, Eds. R. Esser et al (World Scientific, Singapore, 2000).

[18] F. Brochard-Wyart, H. Hervet, and P. Pincus, Europhys. Lett. 26, 511 (1994); F. BrochardWyart, Europhys. Lett. 30, 387 (1995); Y. Marciano and F. Brochard-Wyart, Macromolecules 28, 985 (1985).

[19] G. S. Grest and K. Kremer, Phys. Rev. A 33, 3628 (1986).

[20] P. Ahlrichs and B. Dünweg, J. Chem. Phys. 111, 8225 (1999). 